\title{
Atopiebahnung schon im Mutterleib
}

\section{Die Mutter prägt das Atopierisiko des Kindes stärker als der Vater. Ein Grund ist offenbar der Allergenkontakt der Mutter während der Schwangerschaft, wobei die Allergenkonzentration wie auch der Zeitpunkt des Allergenkontaktes eine Rolle spielen.}

Das Atopierisiko wird bekanntlich z.T. vererbt. Verschiedene Studien zeigen aber auch, daß das Atopierisiko der Kinder bei rund $30 \%$ liegt, wenn die Mutter Atopikerin ist, dagegen nur bei 20\%, wenn der Vater Atopiker ist. Naheliegend ist deshalb, daß Einflüsse auf die Mutter während der Schwangerschaft ihren Beitrag zum Atopierisiko der Kinder leisten.

Eine Untersuchung, die J. Warner, Southampton/Großbritannien, auf dem diesjährigen Kongreß der European Academy of Allergology and Clinical Immunology in Brüssel vorstellte, liefert zu dieser Hypothese überraschende Befunde.

Danach scheint es für das Ungeborene besonders ungünstig $\mathrm{zu}$ sein, wenn die Mutter in der Gravidität einer mittelschweren Allergenexposition ausgesetzt ist, während sich

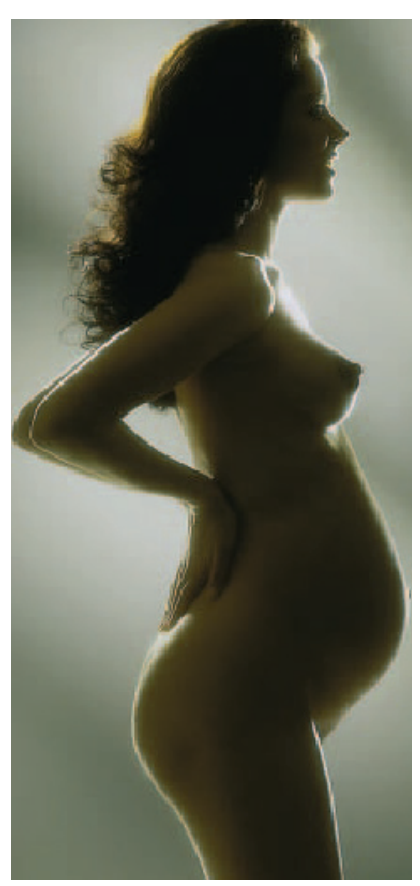

Ist die Mutter während des vierten bis sechsten Schwangerschaftsmonats Allergenen in mittelstarker Konzentration ausgesetzt, ist das Allergierisiko der Kinder besonders hoch. eine besonders hohe

oder gar keine Allergenexposition eher protektiv auswirken.

Auch der Zeitpunkt der Allergenexposition der Mutter ist entscheidend. Eine sensible Phase scheint der Zeitraum zwischen dem vierten und sechsten Schwangerschaftsmonat $\mathrm{zu}$ sein. Beide Beobachtungen treffen zumindest auf Sensibilisierungen gegen Bir- kenpollen sowie Katzen- oder Hausstaubmilben zu.

Aber auch bei Nahrungsmittelallergien, z.B. gegen Eier, ergab sich dieser Zusammenhang: Eine sehr hohe oder niedrige Exposition wirkte sich nicht so negativ aus als wenn die spezifischen IgE-Werte der Mutter im mittleren Bereich lagen.

Warum es $\mathrm{zu}$ dieser eigenartigen Dosis-Wirkungs-Relation mit einem kritischen Zeitfenster zwischen der vierten und sechsten Woche während der Gravidität kommt, ist noch nicht geklärt. Der Ansatzpunkt dürfte aber bei der pathologischen TH2-Antwort der Mutter auf ein Allergen $\mathrm{zu}$ suchen sein. Diese T-Zellsubpopulation, die bei Atopikern in unphysiologisch hohen Konzentrationen $\mathrm{zu}$ finden ist, produziert bei Allergenkontakt eine Reihe von Interleukinen, die zu einer vermehrten IgE-Bildung führen. Tatsächlich findet man in der Amnionflüssigkeit atopischer Mütter erhöhte Spiegel dieser Interleukine (z.B. IL-4, -5, -12 und -13). Diese Zytokine sind im Fruchtwasser erst ab der 20. Schwangerschaftswoche nachzuweisen.

$b k$

Vortrag von J. Warner, Southampton/Großbritannien, anläßlich des Kongresses der European Academy of
Allergology and Clinical Immunology, Brüssel/Belgien, 1999.
Mehr Allergien nach Kaiserschnitt?

Zufall oder nicht? Die Zunahme von Allergien korreliert mit der Zunahme von Kaiserschnitten. Daß hier ein Zusammenhang bestehen könnte, vermutete eine finnische Arbeitsgruppe (J. Kero et al., Turku). Sie gingen von der Überlegung aus, daß sich bei Kindern, die durch einen Kaiserschnitt geboren werden, die normale intestinale Mikroflora nicht so schnell entwickelt wie bei vaginal entbundenen Kindern, wodurch sich früh eine Hyperreagibilität gegen verschiedene Allergene ausbilden kann.

Man verglich deshalb die anhand von Fragebogen erhobenen Daten von vaginal $(\mathrm{n}=158)$ und per Kaiserschnitt geborenen $(\mathrm{n}=$ 161) Kindern über eine evtl. Manifestation allergischer Erkrankungen. Außerdem konnte man auf 12/16 Blutproben aus der Nabelschnur sowie Blutproben dieser Kinder 6 Monate nach der Geburt zurückgreifen.

Die Auswertung ergab eine Tendenz zur Häufung allergischer Erkrankungen bei den Kaiserschnitt-Babies. Der Vergleich der Nabelschnurbluttests ergab, daß zum Zeitpunkt der Geburt die per Kaiserschnitt entbundenen Kinder weniger IgA-produzierende Zellen aufwiesen und die Konzentrationen von Interleukin-6, das für eine gesteigerte IgA-Produktion verantwortlich ist, in dieser Gruppe ebenfalls niedriger war. Der Unterschied verschwand allerdings 6 Monate nach der Geburt.

Die Autoren folgern daraus, daß vor allem atopisch vorbelasteten Eltern geraten werden sollte, die Geburt natürlich ablaufen zu lassen, um dem Immunsystem nicht mehr Schaden als unbedingt nötig zuzufügen.

$b k$

J. Kero, et al. (Turku/Finnland), Poster präsentiert andemy of Allergology an Clinical Immunology, Brüssel/Belgien, 1999. 